Wertheim operation are those of Victor Bonney, and are as follows :

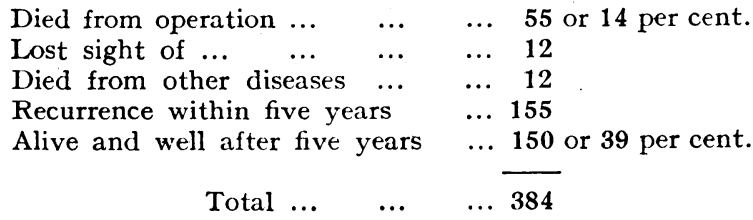

Bonney claims an operability rate of 63 per cent., so his results show a five-years freedom from recurrence of 24.6 per cent. of all cases seen by him. After ten years he has 20 per cent. alive or, if those untraced or dying from other diseases are excluded, 33 per cent. In this country the only figures for radium treatment which can be compared with Bonney's are those of the Marie Curie Hospital, London. Last year's report shows a total of 392 cases treated more than five years ago, and of these 142 , or 36.2 per cent., are alive and well.

In 1934 Beckwith Whitehouse published the five-year results collected from St. Bartholomew's, University College, the Middlesex, and the Marie Curie Hospitals in London, the General Hospital, Birmingham, and the Royal Infirmary, Cardiff. The total number was 507, of which fifty-six patients were alive and well at the end of five years (11 per cent.).

It is easier to decide which is the better method if one operator is able to publish statistics for both operation and radium, since errors dependent upon the personal factor are common to both. One of us (W. F. S.) published his results of the Wertheim operation some years ago, and the cases are still being followed up; at the present time the results are as follows:

$$
\begin{array}{llll}
\text { Died from operation } & \ldots & \ldots & 33 \text { or } 21.4 \text { per cent. } \\
\text { Lost sight of ... } \ldots & \ldots & \ldots & 9 \\
\text { Died from other diseases } & \ldots & \ldots & 5 \\
\text { Recurrence within five years } & \ldots & 48 \\
\text { Alive and well after five years } & \ldots & 59 \text { or } 38.3 \text { per cent. } \\
\text { Total } \ldots & \ldots & \ldots 154
\end{array}
$$

Of those operated upon more than ten years ago there are 120 cases, thirty-three (27.5 per cent.) of which, without making any allowance for those who have died from other causes or are untraced, are alive to-day.

\section{Comparison of Results}

These results are so close to those of Victor Bonneyafter five years 38.3 per cent. alive as compared with his 39 per cent., and after ten years 27.5 per cent. as compared with his 20 per cent. - that we may take them as representative of what can be done with Wertheim's hysterestomy. The only factor which is not comparable is the percentage of cases treated. Working in two large Manchester hospitals, the Royal Infirmary and St. Mary's Hospitals, it was impossible to arrive at an accurate figure without a very laborious search through out-patients' records, but our impression is that our operability rate was less than Victor Bonney's 63 per cent.

The results of our treatment with radium show that 41.4 per cent. were alive and well after five years. Not only is this percentage greater than that obtained with the Wertheim operation, but cases are included of such an advanced character that no operation could have been performed.

Our figures are small. Whether this high percentage of freedom from recurrence will be maintained when we have larger figures remains to be seen, but we are convinced that we are getting better results with radium than we did with Wertheirn's hysterectomy, and therefore we can conscientiously save our patients the pain and dis- comfort of a trying convalescence. This does not mean that we are satisfied; no one can be with figures which show less than half the patients alive after five years.

In one of our hospitals all patients are now being given deep $x$-ray therapy after radium treatment, but this has been in progress for too short a time to permit any comparison. Abroad this combination seems to have given better results. It is also possible that a block dissection of the iliac glands might still further improve matters, though so far we have not been convinced of its efficacy.

While waiting for more efficient methods of prevention or treatment to be evolved we ought to employ the best method at our disposal. The figures which we have been able to put forward strongly support the view that the method is the application of radium, and not the severe surgical procedure still favoured by many gynaecologists in this country.

\section{ACUTE OTITIS MEDIA ITS TREATMENT, WITH RESULTS}

BY

\author{
ANDREW CAMPBELL, M.B., F.R.C.S.ED. \\ EAR, NOSE, AND THROAT SURGEON, GENERAL HOSPITAL, \\ JOHANNESBURG
}

During the course of a common cold, an influenza, or other febrile disease a patient develops an acute inflammation of the middle ear. So long as the otitis remains catarrhal-that is to say, an inflammation without the formation of pus within the middle ear-the treatment is more or less the same: drops to relieve the pain, rest in bed, calomel, heat applied to the ear as radiant heat or in the form of fomentations. Drops to relieve pain may be misleading, as with the cessation of pain we may consider the patient is improving, whereas the contrary is often the case. However, there is no doubt that a considerable proportion of patients respond to the treatment and recover without any further complication.

On the other hand, in a large proportion catarrhal otitis becomes suppurative. There is increasing pain, deafness, tinnitus, vertigo, a rise of temperature-particularly in children-and perhaps even tenderness over the mastoid process. It is now time to consider the advisability of a paracentesis. The drum is usually red and bulging. The bulging alone is a sufficient indication for a paracentesis in the absence of any of the symptoms which have already been mentioned, but usually it is associated with one or more signs or symptoms, such as pain, deafness, and frequently tenderness over the mastoid.

\section{Technique of Paracentesis}

Paracentesis is invariably done in a nursing home under a general anaesthetic such as ethyl chloride. After the skin of the ear and auditory canal is cleaned and painted with iodine the anaesthetic is commenced and an incision is made from below upwards in the posterior half of the drum, care being taken to avoid touching the inner wall of the tympanic cavity with the point of the knife. Pus or serum, sometimes accompanied by gas, escapes. A wick of gauze is introduced as far as the drum, and should be left in till the following morning, when it may be removed.

The discharge may be copious at once, or it may take thirty-six to forty-eight hours before it is free. The discharges are mopped up with sterile wool for forty-eight hours, and then, if copious, the ear is irrigated fourhourly, six-hourly, or thrice daily, according to the quantity of the discharge. The more copious it is the more frequently is the ear irrigated and dried. Irrigation 
which may be carried out with a Higginson syringe fitted with a glass nozzle, should be gentle. It is comforting to the patient, and is tolerated by even the most nervous children. Force should not be used in irrigating.

The essential points in after-treatment are therefore frequent syringing followed by thorough drying of the auditory canal, so that neither debris nor pus may impede the free drainage from the middle ear. Radiant heat is applied to the ear thrice daily for about twenty minutes at a time. The mastoid area may be fomented if there is pain or tenderness in this region. Such symptoms persisting for a day or two after paracentesis do not mean that there is a mastoiditis which will necessitate operation. In the great majority of cases the patient recovers without further surgical treatment. Rest in bed for at least a week is essential after a paracentesis, by which time the temperature in children has usually become normal. Adults have little or no fever with an uncomplicated acute otitis media.

\section{Complications and After-care}

The most serious complication in the early stages of an acute otitis is meningitis, but fortunately this is rare. It is important to watch the temperature and to look for early signs of meningitis if it remains elevated. No rule can be set.down in this matter, as cases vary so much in different seasons and years.

In the uncomplicated case the discharge becomes gradually less in about a week or ten days, and usually ceases in about sixteen days. After the discharge ceases, which coincides with the closure of the perforation or incision in the drum, the hearing is still impaired.

Politzerization daily or thrice weekly will restore the hearing to normal in a fortnight, or in all about thirty days from the commencement of the otitis. This is the course in the average cases. The patient remains in the nursing home for about ten days, and then is treated daily in the consulting room till the discharge ceases, and thereafter twice or thrice weekly till the hearing is restored to normal. In short, the treatment may be summarized as follows: paracentesis, syringe, dry, politzerize, dry, powder.

\section{Value of Syringing}

A considerable amount of confusion exists in South Africa regarding the after-treatment of paracentesis. The treatment which has been described is one I have used for the last fourteen years without variation. A form of treatment frequently described as the " dry treatment" - that is to say, never irrigating, but relying on mopping the canal followed by the instillation of dropshas not been used in this series. If an ear is not syringed it soon collects debris and pus sufficient to interfere with free drainage.

It is idle to say syringing may cause secondary infection of the middle-ear cavity. Assuming the paracentesis and the after-treatment is done aseptically, then we can at worst only drive the same bacteria into the middle ear as came out of it. But we must not overlook the fact that the mucous membrane of the middle ear is acutely inflamed, and little or no cavity exists because of the oedema ; the possibility of driving bacteria back into this almost non-existent cavity is therefore extremely remote.

The use of drops, such as alcohol, magnesium sulphate, glycerin, hydrogen peroxide, picric acid, etc., seems to me to be useless. They cannot penetrate the middle-ear cavity, as the oedematous mucous membrane will not permit this. Moreover, if strong antiseptics such as alcohol, picric acid, etc., did penetrate the middle-ear cleft they would paralyse the ciliated mucous membrano for some hours, and the useful purpose it serves of pro- pelling the discharge onward would be suspended, at least temporarily.

Drops are useful in certain stages ; if a conical perforation develops it is often benefited by ac. boric and alcohol drops, but then there is no danger of the drops reaching the tympanic cavity. It is often said that syringing drives the bacteria into the mastoid process, but the mastoid cells are in an active state of inflammation in every case of acute otitis media. This is well demonstrated in the early stages by $x$ rays, in which the cells show up as cloudy compared with the normal mastoid. Traumatic perforations, however, should not be syringed unless they reach the stage of profuse discharge; then they may be treated as a paracentesis case.

\section{The Dry Method}

The method of mopping up the discharge has been described as the dry method, but I believe the method which I have described is the true dry method. The only wet part of the treatment is the irrigation ; then the canal is dried thoroughly. After the patient leaves the nursing home the ear is syringed daily, dried, politzerized so as to drive away any secretions out of the tympanic cavity, dried again, and finally powdered with boric acid.

A study of the literature on the subject of the treatment of acute otitis has not presented anything very definite ; some syringe, and others do not; no hard-andfast rules have been laid down by either side.

\section{First Series of Cases}

Cases which were paracentesized before spontaneous rupture of the drum took place numbered 339 ; of these, twenty-four cases, or 7.06 per cent., required a simple mastoid operation. One of the paracentesis patients died as a result of pneumococcal meningitis without macroscopic evidence of involvement of the mastoid cells; it seemed to be a fulminating type.

The healing of the perforation took on an average sixteen and a half days, and the return to normal hearing averaged thirty days from the date of paracentesis. The hearing result was noted in only 161 cases, as many small children could not be tested, and others with previous hearing defects could only be estimated. Of the 161 patients all were satisfied that no defect in hearing was left after healing was complete. Age and sex were noted in all cases, but they have no bearing on the subject, except to demonstrate that the majority were children.

\section{Second Series of Cases}

Cases which were paracentesized after the discharge had commenced, owing to pain, elevation of temperature, mastoid tenderness, etc., numbered 126. It is surprising how frequently on incising the drum in such cases pus escapes under pressure. Of this series twenty-two, or 17.4 per cent., required a simple mastoid operation. There were a few cases where the operation was performed two or three days after paracentesis, and I think these should be left out of this calculation, as they probably had a mastoiditis when the paracentesis was done. The more correct percentage, therefore, would be between 14 and 15. There was one death in this series, due to meningitis caused by a haemolytic streptococcus, without macroscopic involvement of the mastoid.

A comparison of the first and second series demonstrates that early paracentesis, before spontaneous rupture takes place, is the best form of treatment. Quite apart from an early paracentesis there are other factors which influence the disease, the chief of these being, I believe, the virulence of the infection and the resistance of the patient. In certain years the disease is mild, in others 
it is exceptionally virulent, and liable to give rise to complications.

The most frequent causes of otitis media in these cases were the common cold and influenza, followed in order by tonsils and adenoids, scarlet fever, and measles. Nine cases followed operation on the nose, of which four required mastoid operations.

\section{Cases Requiring Operation}

One hundred and six patients were operated on by the Schwartze method, which is commonly known as the simple mastoid operation, with preservation of the drum and return of hearing in the vast majority of cases to normal. As a routine method the mastoid antrum, the lateral sinus wall, and the dura of the middle fossa were exposed, while the mastoid tip was explored and removed in most cases. Except in a few isolated instances the routine has been adhered to, so that the possibility of overlooked infected cells, a perisinusitis, or extradural abscess was minimized.

Twenty-two, or 20 per cent., of these patients had been paracentesized before spontaneous rupture; the balance of 80 per cent. were either not paracentesized at all or only after spontaneous rupture had occurred. These figures show the importance of early paracentesis; 93 per cent. of cases of acute otitis media are cured by paracentesis before rupture, with return of hearing to normal within a month.

After the Schwartze operation is completed an attempt is made to syringe saline through the aditus so that it comes out of the perforation and into the external auditory canal. Care must be taken not to force the liquid through in any manner. The cavity is then filled up with absolute alcohol, dried, iodoform gauze packing introduced, and the incision is partly closed. The external canal is plugged with narrow iodoform gauze, so that no narrowing of the canal is allowed to take place. The wound is left undisturbed for four days, when the packing is removed, which is always a painful process. Fresh gauze is reintroduced daily till the cavity heals by granulation. The perforation usually heals in a few days, and from then on the cavity is allowed to heal up rapidly. A plastic operation to bring the skin edges together is sometimes necessary. Eventually politzerization will restore hearing to normal. There should be no mortality in uncomplicated cases of acute mastoiditis.

\section{Results of Operation}

In the 106 patients operated on the ages varied from 6 months to 60 years ; 48 per cent. were under the age of 12. The hearing result was noted in sixty-seven cases, of which fifty-eight were normal, four were noted as good, and five as moderate. It must be remembered in this connexion that patients with indifferent hearing before operation were satisfied that they heard as well after operation.

The perforation healed completely in 102 out of 106 cases. A permanent perforation remained in three cases, and there was no note in one. The dura of the middle fossa was exposed in all but two cases, both under the age of 1 year. The lateral sinus was exposed in all but one case, again under 1 year of age. A perisinal or extradural abscess was present in nine cases.

Temporary facial paralysis developed in three cases, lasting six to nine months before recovery. In all these cases the cells dipped medial to the facial canal.

There were five cases of Bezold's mastoiditis, but they did not require any further intervention beyond removal of the mastoid tip. Only one case of Gradenigo's symptom-complex was observed.
The time of healing varied from eleven to 150 days, the average being thirty-five days ; plastic closure was necessary in twenty-six out of 106 cases, chiefly to prevent unsightly post-auricular depression.

The mastoid process was cellular in, eighty-three cases, diploic in fourteen, sclerotic in one case (a diabetic), and there is no note in eight cases.

Blood-clot healing was the method employed in thirteen cases early in the series. The mastoid cavity, after being thoroughly cleaned by syringing and alcohol, was allowed to fill up with blood and the wound closed. Nine of these recovered without breaking down of the wound. The balance recovered after breaking down or reopening of the wound. I do not recommend this method. Within the last eighteen months fifteen cases have been drained through the post-auricular wound by means of one or more rubber tubes. The wound is closed above and below the tubes. After four days the tubes are removed painlessly and gauze packing substituted daily. By this method there is no pain during the dressings, and the results are as good as the packing method, and I believe a little quicker in healing.

Two of the 106 cases subsequently required a radical mastoid operation.

\section{Mortality}

There were two deaths in this final series of 106 cases. Both occurred in cases of long-standing mastoiditis, which should have been operated on very much earlier. Both had diploic mastoids; one died of pneumonia, and the other of cellulitis of the neck, followed by meningitis. This is not to say that I have not had other deaths following mastoid operation, but these have all been in cases where intracranial complications were present , beforo operation, and such cases are not included in this series.

\section{Conclusion}

The main purpose of this article has been to give the results of one method of treatment of acute suppurative otitis media and its most frequent complication-acute mastoiditis. Early paracentesis is strongly indicated to prevent so far as possible the onset of mastoiditis and ensure a quick convalescence. Only 7 per cent. of patients who have had an early paracentesis require a mastoid operation. Except under exceptional circumstances an acute mastoiditis does not develop for seven to twelve days after the onset of an otitis.

No attempt has been made to tabulate indications for operation, as no hard-and-fast rules can be laid down. Some patients have mastoiditis without any discharge, others with; some with swelling, others without; while tenderness and a high temperature may exist or not.

Experience counts as much here as in any other field of surgical work, and even then a furuncle may be mistaken for a mastoiditis.

To commemorate the many and great contributions made to the surgery of the bones for more than forty years by Dr. Albin Lambotte, a jubilee book is to be compiled and presented to him. It will be recalled how much the world owes to his operative technique for the open method for the reduction and setting of fractures and his procedure of metallic prosthesis. The book will be limited to articles by authorities on the surgery of the bones and joints, and among the contributors will be Mr. H. A. T. Fairbank and Professor Hey Groves of this country, and Drs. Albee and Smith-Petersen of the United States. The articles will be published in French, Dutch, English, German, or Spanish. It will appear in one or two volumes, fully illustrated, and the cost will be 30 belgas. Applications for it should be addressed to Dr. Jean Verbrugge, 75, Avenue van Ryswyck, Antwerp. 